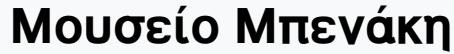

Tóp. 1 (2001)

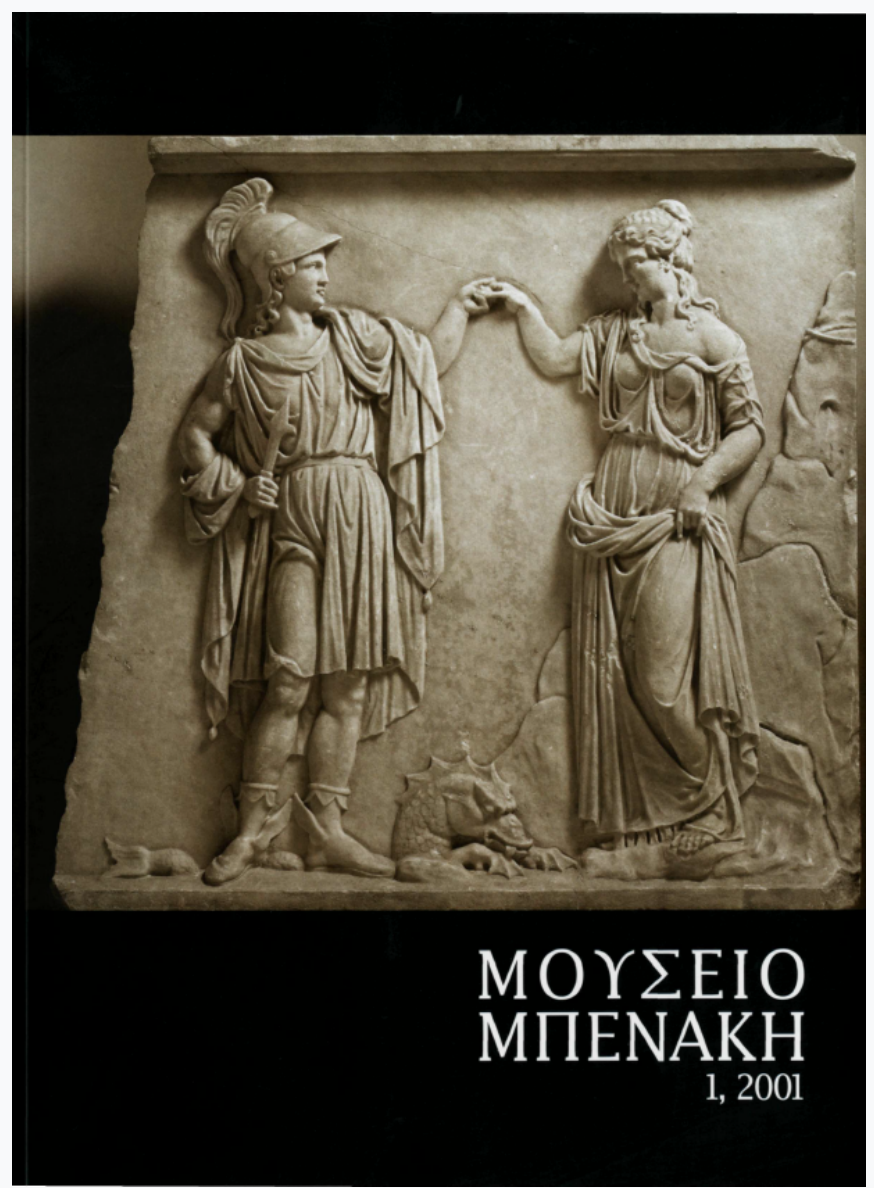

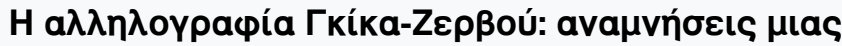

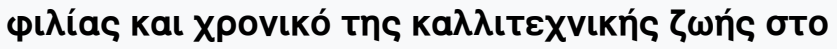

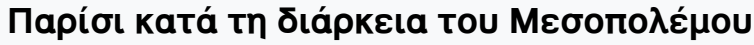
(1933-40)

Jean-Pierre De Rycke

doi: $\underline{10.12681 / \text { benaki.18333 }}$

Copyright @ 2018, Jean-Pierre De Rycke

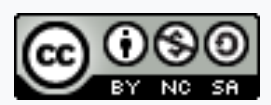

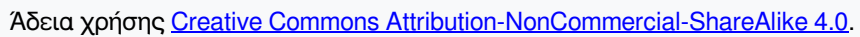

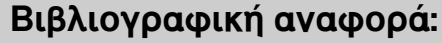

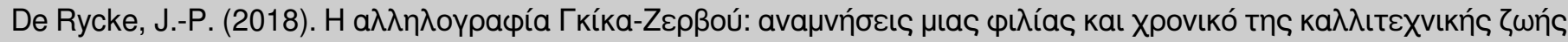

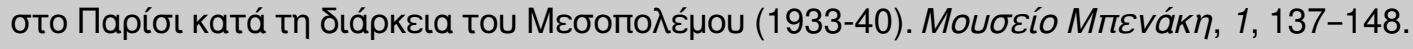

https://doi.org/10.12681/benaki.18333 


\section{La correspondance Ghika-Zervos: souvenirs d'une amitié et chronique de la vie artistique parisienne durant l'entre-deux-guerres (1933-40)}

Au sein DeS ARCHIVES de la Pinacothèque Ghika, la correspondance entre le peintre Nico Ghika et Christian Zervos (1889-1970), un Grec d'origine qui fut un des principaux critiques d'art et animateurs de l'avantgarde parisienne à l'époque du modernisme, occupe une place intéressante (fig. 1). Ce fonds représente environ soixante-dix lettres rédigées en français et qui couvrent une assez longue période s'étendant approximativement de 1933 à 1970, année du décès des époux Zervos. $^{1}$

En réalité, il faudrait plutôt parler de correspondance entre "les" Ghika et "les" Zervos puisqu'il s'agit en effet d'un échange croisé de lettres entre le couple Christian et Yvonne Zervos d'une part, et celui formé par Nico et Tiggie Ghika d'autre part.

Comme le veut la règle, les lettres conservées à Athènes sont naturellement celles que les émissaires parisiens -Christian ou Yvonne Zervos- adressaient à leurs correspondants en Grèce -Nico ou Tiggie. Et ce n'est qu'exceptionnellement qu'on rencontre dans ces documents l'un ou l'autre brouillon de réponse de l'un des destinataires à ses amis parisiens. Il s'en suit logiquement que le contenu des lettres concerne en priorité le propre vécu des Zervos en France, tout en l'assortissant accessoirement d'attentions ou de sollicitations diverses à l'égard des Ghika.

Pour l'essentiel, la correspondance adressée par les Zervos aux Ghika a trait à l'activité éditoriale de Christian Zervos, pour laquelle ce dernier sollicita plus d'une fois l'appui logistique de son compatriote Nico en Grèce. Une partie du courrier concerne également la promotion de l'oeuvre personnelle de Ghika dont Zervos fut un des principaux soutiens ainsi que l'un des plus fervents admirateurs. Mais c'est surtout en tant que chronique "quotidienne" de la vie artistique parisienne que le recueil épistolaire représente un intérêt particulier.

Le moment précis de la rencontre entre Zervos et Ghika reste en partie un point d'interrogation. Selon Zervos lui-même, mais qui fait probablement erreur, ce serait en 1926 - année de création de sa revue les Cahiers d'art - qu'il aurait fait la connaissance de Nico à Paris où le jeune artiste né en 1906 essayait alors de s'imposer. Selon N. Petsalis-Diomidis, elle remonterait au contraire à $1924 .^{2}$ Ce qui semble toutefois certain, c'est que les deux hommes se connaissaient déjà en 1925 comme le prouve une photographie de groupe prise cette année-là à Paris dans les Jardins du Luxembourg, et où l'on aperçoit les deux protagonistes en compagnie d'autres amis d'origine grecque comme A. Katakouzinos, Tériade, ${ }^{3}$ Katsimbalis ainsi que le sculpteur Tombros, futur fondateur de la revue athénienne d'avant-garde 20os aionas (fig. 2).

Qui était donc Christian Zervos? Né à Argostoli dans l'île de Céphalonie, le 1er janvier 1889 - et décédé à Paris en 1970 -, son enfance et sa première jeunesse se déroulent à Alexandrie. Après quelques années passées à Marseille, il s'installe à Paris en 1911 pour terminer ses études en Sorbonne où, pendant la guerre 1914-18, il défend sa thèse de doctorat des lettres consacrée au philosophe platonicien byzantin, Michael Psellos. Il s'investira par la suite dans une activité éditoriale et 

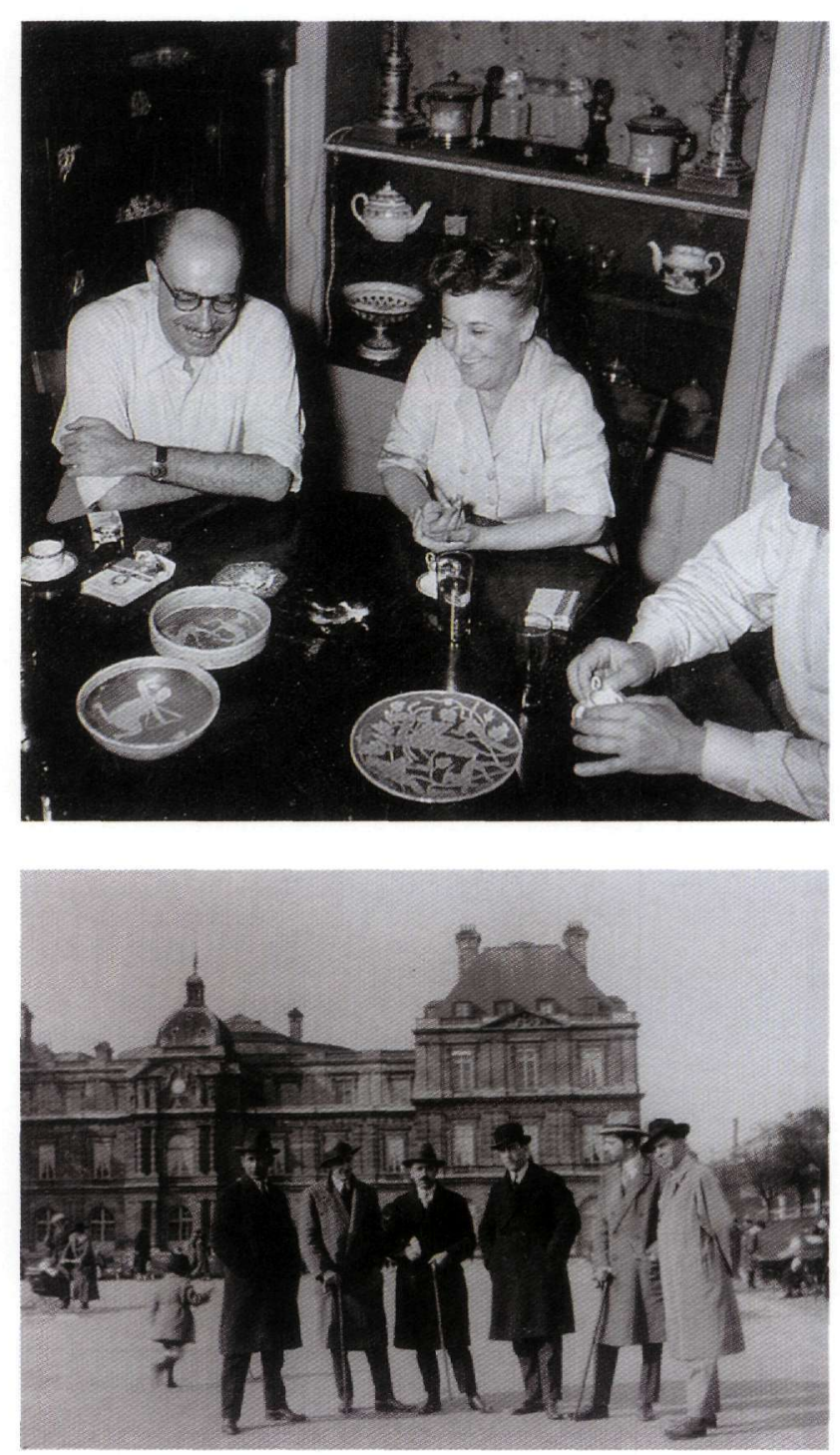

Fig. 1. Le couple Ghika avec Christian Zervos (à droite). Athènes 1941(?). Musée Benaki, Pinacothèque Ghika.

Fig. 2. Paris, Jardins du Luxemburg, 1925. De gauche à droite: Ch. Zervos, A. Katakouzinos, Tériade, Katsimbalis, Ghika et Tombros. Musée Benaki, Pinacothèque Ghika.

dans la promotion de l'art moderne dans la capitale française, en collaboration avec sa femme Yvonne. La revue Cahiers d'art, qu'il fonde en 1926, et la galerie du même nom qui l'accompagne à la rue du Dragon formeront un des fers de lance de l'avant-garde artistique parisienne dans l'entre-deux-guerres. ${ }^{4}$

La correspondance entre Ghika et Zervos conservée dans les archives de la Pinacothèque démarre véritablement en 1933-34. Cette période coïncide en effet avec le moment où Ghika et sa femme délaissent progressivement Paris pour se réinstaller définitivement en Grèce. Plusieurs lettres des Zervos feront d'ailleurs état de cette situation pour la déplorer et pour implorer Nico Ghika de revenir s'établir dans la capitale française qui lui ouvrirait les portes du succès. ${ }^{5}$

C'est également l'époque où Zervos achève la préparation de ce qui constituera son oeuvre littéraire majeure: une monumentale histoire de L'Art en Grèce publiée pour la première fois en 1934 et qui fera l'objet de nombreuses rééditions. ${ }^{6}$ Ghika est alors sollicité par Zervos pour rassembler sur place une partie de la documentation nécessaire à son ouvrage. ${ }^{?}$

Le point de vue de ce livre est original. Comme le rappelle Zervos lui-même dans la préface de ce dernier, la vision traditionnelle de l'art grec avait jusqu'alors été tronquée par la présentation abusivement "classicisante" qu'en avaient donée les historiens d'art des générations précédentes.

Ce principe provoqua dès la fin du $19 \mathrm{e}$ siècle le rejet de l'art grec par les artistes qui, combattant l'académisme, voulaient retourner aux sources de l'art. Ainsi Gauguin dénonçait-il le modèle grec comme le pire ennemi des créateurs soucieux d'authenticité.

Le propos de Zervos est de montrer que, à côté de la production classique de l'âge d'or et de l'époque tardive hellénistique, il existe un art grec plus ancien qui répond davantage aux critères de "fraîcheur", de jeunesse et de pureté que recherchent les tenants de l'avant-garde. Et dans ce contexte particulier, le groupe cycladique avait une valeur exemplaire.

C'est tout ce patrimoine "archaïque" ou "primitif", alors relativement méconnu de la Grèce, qui peut, selon Zervos, jouer le même rôle d'inspiration pour les artistes contemporains que les arts dits "sauvages" d'Afrique ou d'ailleurs, tels qu'ils encouragèrent la rénovation de l'art occidental dès les premières expériences cubistes et fauvistes au début du $20 \mathrm{e}$ siècle.

Le grand dessein de Zervos était d'ailleurs de réconcilier l'ancien et le présent, comme il l'exprimera clairement dans un texte de présentation des Cahiers d'Art qui paraît en 1932: “... Cahiers d'art montre au jour le jour le développement de l'oeuvre des meilleurs parmi les artistes d'aujourd'hui; et étudie les civilisations du passé en vue de montrer l'unité de l'esprit humain, sous la complexité de ses apparences, la modernité des grandes oeuvres 
anciennes et les équivalences entre ces oeuvres et celles d'aujourd'hui.».

La modernité de la démarche de Zervos à travers l'édition de son livre sur l'art en Grèce est renforcée par la circonstance qui lui permit de l'entreprendre ainsi que l'auteur le révèle dans sa dédicace introductive: le IVème Congrès International d'Architecture Moderne qui se tint à Athènes en 1933.

Cette occasion particulière permit en effet le rassemblement de quelques personnalités emblématiques de la création artistique et littéraire occidentale d'avant-garde sur le sol grec. Le peintre Fernand Léger, l'architecte Le Corbusier, le poète Gaston Bonheur ou le critique Maurice Raynal participèrent entre autres à cette manifestation internationale en compagnie de Zervos et ce fut là l'occasion pour Ghika de les rejoindre ou de les recevoir personnellement dans son fief insulaire d'Hydra, comme nous le montrent encore quelques photographies d'époque (fig. 3).

A leur tour, ces différents acteurs de la vie littéraire ou artistique livreront leurs propres impressions sur la réalité culturelle de la Grèce ancienne et moderne au sein du volume de Zervos, regroupées sous le titre Témoignages de poètes, peintres, architectes et écrivains d'art contemporains. On y rencontre les contributions de Pierre Guéguen, Fernand Léger, Le Corbusier, Gaston Bonheur, Ozenfant et Maurice Raynal.'

L'Art en Grèce paraît en janvier 1934. La première édition se révèle apparemment une très mauvaise affaire comme le révèle une lettre adressée par Zervos à Nico Ghika en 1934 et dans laquelle il reproche ni plus ni moins au libraire Kauffmann d'Athènes de ne pas respecter ses engagements. Zervos craint même un moment pour la survie des Cahiers d'art, tant sa situation financière est compromise. ${ }^{10}$

Au-delà de ce problème pécunier, les craintes de Zervos pour sa revue sont peut-être également justifiées par la concurrence toute récente d'une autre revue, le célèbre "Minotaure", fondée en 1933 par son ancien collaborateur Tériade en compagnie d'Albert Skira.

Grec d'origine tout comme lui, Tériade, de son vrai nom Efstratios Eleftheriades (1897-1983), a quitté les Cahiers d'art, selon ses propres termes "pour fonder une nouvelle revue avec l'intention d'y inclure la participation des surréalistes, de réagir contre le cubisme puriste, de brasser des idées et d'intégrer les arts plastiques à la poésie,

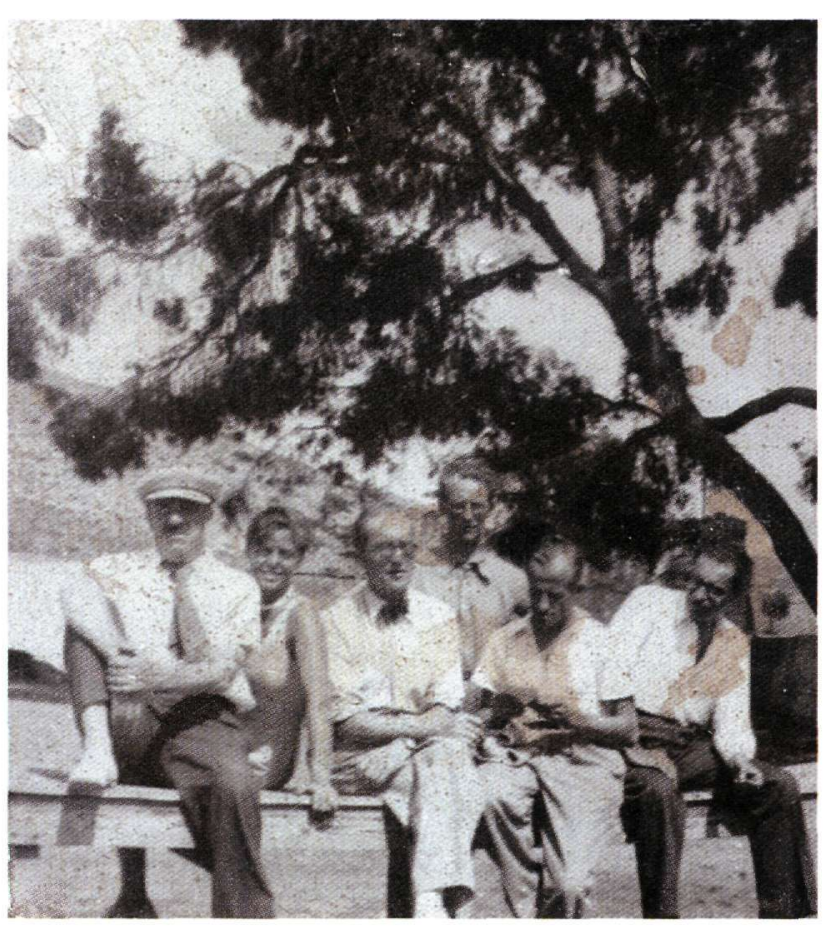

Fig. 3. Hydra(?) 1933. De gauche à droite: Fernand Léger, (?), Le Corbusier, (?), (?), Ghika réunis à l'occasion du IVème Congrès International d'Architecture Moderne. Musée Benaki, Pinacothèque Ghika.

la philosophie, la psychanalyse, la littérature, l'ethnologie et même la musique». ${ }^{11}$

Minotaure entend donc couvrir un champ plus large que les Cahiers d'art de Zervos et ce nouveau principe n'est peut-être pas étranger à l'introduction par ce dernier d'une série d'articles consacrés au surréalisme dans sa publication, dès les premiers numéros de $1935 .^{12}$

Sur le plan de la vie civile, 1934 est également l'année où éclate en France l'affaire Stavisky qui débouchera sur les sanglantes émeutes de février 1934 et mettra même la République en péril. Face à la corruption présumée de plusieurs membres du parti radical, la droite mobilise ses troupes et provoque la chute du gouvernement Chautemps, aussitôt remplacé par Daladier. Le soir du 6 février, une manifestation de masse décrétée par toutes les organisations de droite, ainsi que le parti communiste, tourne mal. Echanges de coups de feu. L'émeute devient générale. Le matin du 7 février, on dénombre 15 morts et 1500 blessés. Paris est traumatisée. $^{13}$ 
Christian Zervos fait directement allusion à ces événements dans une de ses lettres à Ghika: «... Ici tout est mort... terriblement mort. Ni expositions, ni intérêt, rien du tout. Tout le monde est plongé dans le cafard jusqu'aux cheveux, c'est quelque chose d'atroce. Surtout depuis les derniers évévements [émeutes de février 1934] tout est en panne, on croirait un pays en agonie. Vous ne pouvez vous imaginer depuis notre retour comme tout est changé. Paris est un immense village sans joie, sans vie nocturne. Je ne pouvais m'imaginer qu'un peuple aussi vivant pouvait se laisser aller aussi profondément dans le désespoir. Heureusement que nous avons l'affaire Stavisky pour nous donner des émotions, sans quoi tout serait terne.».

Et Zervos de dissuader son correspondant de rentrer en France à ce moment: "Je crois que vous feriez bien de ne pas venir avant la fin Mars. Il n'y a rien à faire et vous ne perdez rien en restant en Grèce, au moins vous conservez intact votre enthousiasme...... ${ }^{14}$

Dans la même lettre, Zervos demande à Ghika de lui apporter quelques reliefs dont il a déjà montré certaines reproductions autour de lui, lesquelles ont reçu un accueil favorable. Il s'agit de la série des bas-reliefs géométriques que Ghika entreprend en 1934 et dont certains spécimens seront exposés la même année à la Galerie des Cahiers d'Art, où les oeuvres de l'artiste grec côtoient les réalisations d'autres créateurs en vogue de cette époque comme Hans Arp, Jean Hélion ou Sophie Tauber-Arp. Quelques clichés conservés dans les archives photographiques de la Pinacothèque Ghika révèlent encore les arrangements de cette exposition où les reliefs de Ghika voisinaient avec différentes compositions de Arp ou une peinture abstraite de Jean Hélion (fig. 4). ${ }^{15}$

Pendant ce temps, Yvonne, l'épouse de Zervos (née Yvonne Marion, Paris 1905-ibid. 1970), ne demeure pas en reste. Très active également dans la promotion de l'art moderne, elle-même créatrice à ses heures, elle fonde sa propre société de "design" en 1935, Stylclair, qui regroupe les noms de collaborateurs aussi prestigieux que Aalto, Aartaria, J. Bossu, Breuer, Gropius, Hafeli, Hubacher, Kienzle, Le Corbusier - Pierre Jeanneret et Perriand, Merkelbach, Moser, Rietveld, Roth, Stam, Steiger, Thomas. ${ }^{16}$

C'est également l'époque où les Zervos entretiennent des liens d'amitié avec une grande partie de l'élite artistique parisienne. "...Samedi et dimanche, nous sommes allés avec les Kandinsky à Dieppe. C'était très joli...». ${ }^{17} \mathrm{Et}$ plus loin dans la même lettre: Les Laurens [le sculpteur Henri Laurens (1885-1954) et sa femme Marthe], «je les aime de plus en plus; pour eux, la vie est aussi un miracle». ${ }^{18}$

Mais le partenaire privilégié des Zervos est sans conteste Pablo Picasso que le couple fréquentera assidûment jusqu'à la fin de leur vie, et à qui ils consacreront la plus grande part de leur activité inlassable pour la promotion de l'art moderne (fig. 5). ${ }^{19}$

A ce moment, les Zervos habitent à l'hôtel, où ils ne peuvent se "coucher avant deux trois heures du matin. Nous traînons aux "Deux Magots", chez "Lipp"] deux des établissements parisiens les plus célèbres et les plus fréquentés par les artistes à Saint-Germain-des-Prés durant l'entre-deux-guerres] jusqu'à des heures impossibles. Je suis très heureuse de cette nouvelle vie, précise Yvonne, nous voyons des gens qui nous apportent toujours du nouveau. ${ }^{20}$

Mais elle regrette en même temps l'absence des Ghika qui demeurent toujours en Grèce à ce moment. «...Je regarde souvent votre atelier qui est toujours noir et cela me désespère!!!». ${ }^{21}$

Signalant la préparation d'un numéro spécial des Cahiers d'art sur Picasso, elle révèle à Tiggie que ce dernier "fait des poèmes très beaux! Car il divorce avec sa femme [la Russe Olga Khokhlova]. Depuis 6 mois, il ne peint plus, il fait seulement des poèmes... et maintenant nous le voyons quelquefois aux "Deux Magots".). ${ }^{22}$

Picasso vit alors en effet ce qu'il appellera lui-même plus tard «la pire époque de sa vie». En cet hiver 193435 , sa femme Olga vient de quitter le domicile conjugal en emmenant leur fils Paulo, mais elle refuse le divorce. Pendant ce temps, le désarroi du peintre est renforcé par la grossesse de sa maittresse Marie-Thérèse Walter qui le rend à la fois fou de joie et complètement désemparé, n'ayant personne à qui se confier car il veut cacher la nouvelle.

Picasso ne travaille plus. Alors, il écrit. Comme le dit son biographe Pierre Cabanne, "il faut bien qu'il s'exprime d'une manière ou d'une autre. Ayant cessé de peindre, il prend la plume.». ${ }^{23}$

Les réactions de l'entourage et des proches de l'artiste sont mitigées par rapport à cette nouvelle occupation. "On me dit que tu écris. De toi, je crois tout possible», lui dit sa mère dans une lettre. "Si un jour on me raconte que tu as célébré la messe je le croirai aussi.». ${ }^{24} \mathrm{La}$ réaction de 

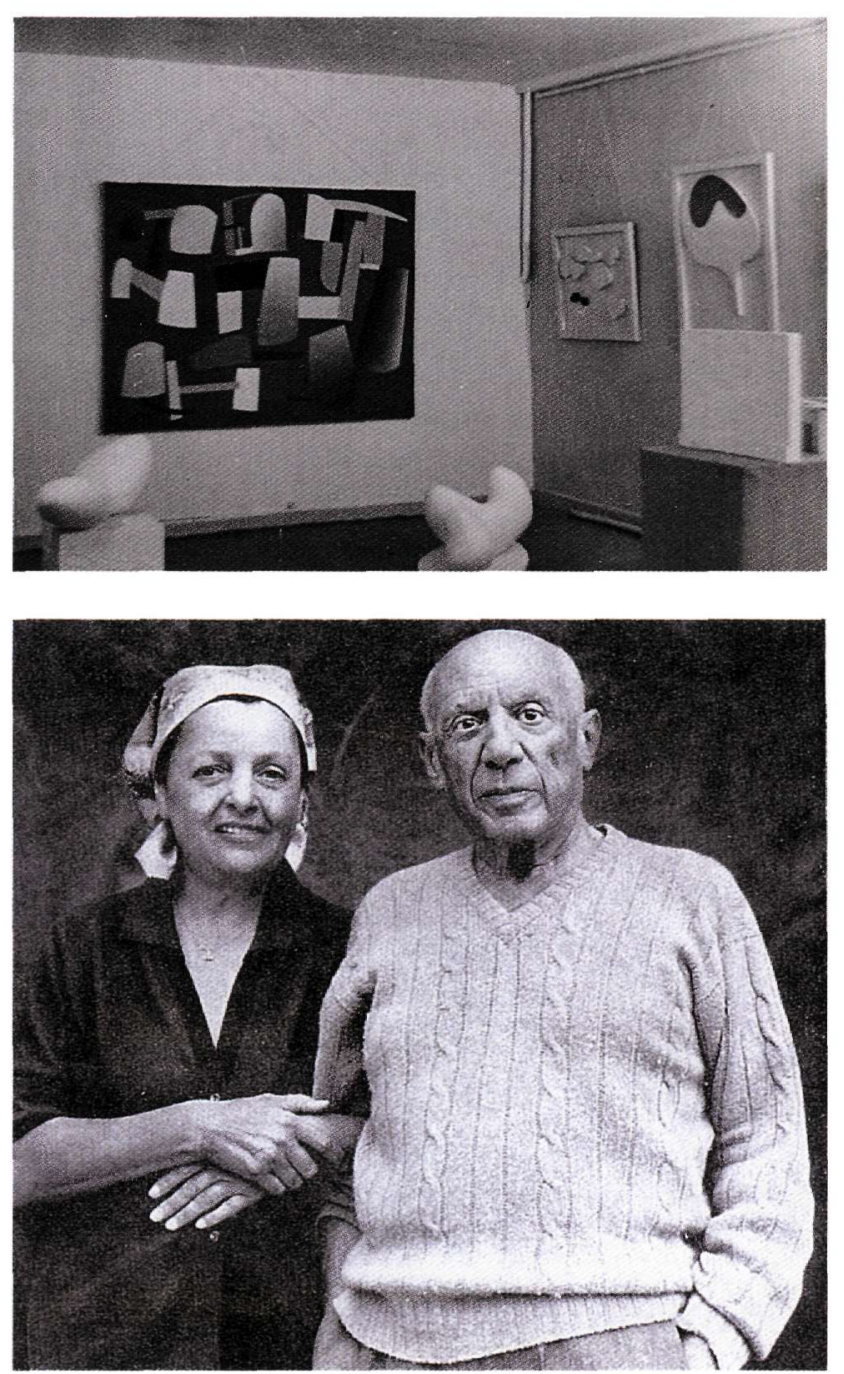

Fig. 4. Paris, Galerie des Cahiers d'Art, 1934. Oeuvres de Ghika, Jean Arp et Jean Hélion (Musèe Benaki, Pinacothèque Ghika).

Fig. 5. Yvonne Zervos et Pablo Picasso en 1968 (N. Had-

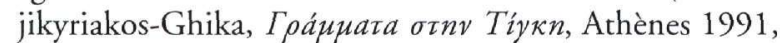
fig. 33).

Gertrude Stein est différente, qui supporte mal «la stérilité des peintres dans la poésie.». Elle reproche à Picasso "d'avoir trop regardé les dessins de Cocteau qu'elle jugeait "plus choquants que des dessins simplement mavais" et d'agir de la même manière dans ses poèmes "qui sont plus choquants que des poèmes simplement mauvais."..".

Mais le malaise particulier de Picasso semble rencontrer celui plus général de l'art qui rentre peu à peu dans une impasse. Alors que la République française se remet difficilement d'une crise qui a failli lui coûter son existence [les émeutes de février 1934], c'est toute la création dans son ensemble qui stagne au moment où retombe la colère surréaliste. Crise de civilisation? Le mot est à la mode. W. George publie alors sa Destruction de l'humain, un pamphlet réactionnaire dans lequel il veut remettre en selle l'humanisme, les valeurs sensibles, le durable, l'éternel; de son côté, E. Faure décrit une Agonie de la peinture dans L'Amour de l'Art. ${ }^{26}$

Il est vrai peut-être aussi que les héros commencent à être fatigués. Comme elle le confiera un peu plus tard dans une lettre de 1938 où elle reproche une nouvelle fois aux Ghika de ne pas revenir tenter leur chance à Paris, Yvonne Zervos déplore le manque de renouvellement de la création dans la capitale en cette fin des années trente. "...Vous savez qu'il n'y a plus un seul jeune peintre ici, car Miro [né en 1893] ne peut plus être considéré comme jeune, ayant plus de 44 ans. ${ }^{27}$ Quant à Picasso, né en 1881 et alors âgé de 57 ans, il ne doit plus vraiment être considéré comme un prophète.

«...Cela me fait penser que vous avez tort, mille fois tort de ne pas venir à Paris. [...] Nico aurait déjà fait son chemin ici. L'autre jour Gallatin du Museum of Living Art admirait le petit tableau de Nico que nous avons à l'appartement et voulait faire sa connaissance. Dites-moi franchement pourquoi vous ne venez pas à Paris, au moins pendant six mois de l'année. Nico n'est pas encore oublié.... ${ }^{28}$

Mais la seconde partie des années trente avance aussi le spectre de la guerre qui se profile un peu partout à l'horizon. La tragédie commence en Espagne avec la guerre civile de 1936-39. Les Zervos sont des témoins privilégiés du conflit, avant-coureur des désastres futurs. En effet, Christian prépare alors une série d'ouvrages consacrés à la péninsule ibérique: L'Art de la Catalogne [1937] et Les oeuvres du Gréco en Espagne [1939].

Et les Zervos n'hésitent pas à se rendre plusieurs fois sur place pour rassembler la documentation. Parfois au péril de leur vie. Plusieurs lettres font allusion aux risques encourus..$^{29}$ Mais au-delà du danger, ces séjours en Espagne sont également une source de bonheur. «... Je prépare un ouvrage sur l'art médiéval en Catalogne. Je viens de parcourir toutes les provinces de la Catalogne. La vie est ici merveilleuse», s'enthousiasme Christian Zer$\operatorname{vos}^{30}$ Et sa femme de préciser qu'avant leur départ pour l'Espagne, ils étaient bien fatigués et découragés. 
"Ce voyage nous a donné tellement de joie et d'espoir que maintenant nous avons des forces pour travailler et entreprendre beaucoup de choses..... ${ }^{31}$

Un des objectifs de l'ouvrage sur L'art en Catalogne est également d'ordre politique. Il s'agit par là de dénoncer la propagande nationaliste qui tentait de reporter la responsabilité des dégâts occasionnés au patrimoine artistique catalan sur les Républicains. «... Taky [Christian Zervos] a fait un très beau livre qui servira à prouver que tous ces "chefs d'oeuvre" n'ont pas été brûlés [par les Républicains] et que tout est en parfait état». ${ }^{32}$

Quant au livre sur le Greco, il semble particulièrement faire la fierté de son auteur. "...En ce moment, je prépare un livre vraiment formidable sur le Gréco (sur les tableaux authentiques du Greco, car les livres parus récemment sur lui sont pleins de faux dans la proportion de $65 \%$ et plus), confie-t-il à Nico Ghika. ${ }^{33}$ Outre qu'il est beau, ce livre m'est très cher car il m'a donné l'occasion de visiter l'Espagne - Madrid, Valence, Barcelone en pleine guerre civile - et que nous avons failli d'un rien y laisser notre peau. [...] J'aimerais que vous lisiez mes textes sur le Greco parce que j'étudie sa peinture du vrai point de vue: plastique et mystique.". ${ }^{34}$

Le poète espagnol Rafael Alberti gardait encore en 1971 le souvenir de cette époque héroïque. "Yvonne Zervos, très jeune et très jolie, arrive avec Christian Zervos à Madrid, à l' "Alliance des Intellectuels". Je crois que c'était à la fin de 1936, après les jours les plus graves et les plus glorieux de la défense de la capitale [...]. Picasso avait déjà été nommé directeur du Musée par le gouvernement de la République: le 1er directeur au monde d'un musée ambulant, puisqu'en peu de temps, il dut passer de Madrid à Valence, de Valence à Barcelone et de Barcelone à Genève...»."35

"L'arrivée d'Yvonne et Christian Zervos coüncida avec les jours les plus durs et les plus fameux de notre lutte. Maria Teresa et moi les accompagnâmes à travers les rues et les quartiers excentriques proches du front. Nous montâmes à la "Telefonica", alors l'un des édifices les plus élevés de Madrid. De là, ils virent, impressionnés, les destructions causées par les bombardements, les blessures sanglantes de la ville [...]. Yvonne à Madrid, prit intérêt à tout, s'informant de la progression du sauvetage des collections particulières situées dans des endroits exposés, rendant visite à la Junte pour la sauvegarde du trésor artistique...». ${ }^{36}$

"Pendant ce temps, Christian Zervos vivait la passion du
Gréco, son illustre compatriote. Il étudiait et expertisait les oeuvres du peintre, que l'on venait de découvrir dans les églises situées à proximité du front et de mettre à l'abri [...]. Yvonne nous parla, encore émue, de son enrôlement dans les équipes catalanes chargées de réunir au Musée d'Art de Catalogne les objets trouvés dans les églises..... ${ }^{37}$

A la même époque, Nico Ghika s'intéresse de son côté à l'art dramatique. En 1937, il dessine le décor et les costumes de As you like it de Shakespeare qui représente semble-t-il le premier décor "moderne" en Grèce. ${ }^{38}$ Sans doute requiert-il à cette occasion la collaboration d'Yvonne Zervos comme il apparaît dans une lettre de réponse que celle-ci adressa au peintre le 1er janvier 1937: «...En rentrant d'Espagne, j'ai trouvé également ton autre lettre qui me demandait des patrons pour robes? Est-ce encore temps?...". ${ }^{39}$

Une autre lettre de Christian Zervos à Nico Ghika apporte quelques informations à ce dernier qui s'interroge sur l'actualité théâtrale parisienne en 1939: «...Ici aussi, il y a quelques tentatives de renouveler le théatre. C'est l'acteur Barrault [Jean-Louis Barrault (1910-1994)] qui s'en occupe. Il vient de monter l'Hamlet de Laforgue et une féerie tirée de la Faim de Knut Hamsun. C'est tout ce qu'il $y$ a de nouveau au point de vue théatre...»."

En même temps, les Zervos continuent de soutenir la peinture de Ghika qui sollicite toujours leur appui à Paris. "... Yvonne et moi nous pourrions organiser une exposition de vos oeuvres à la Galerie Pierre $e^{41}$ qui est devenue la meilleure de Paris du point de vue de la qualité (Picasso, Léger, Matisse, Braque, Miro et un peintre nègre que nous venons de découvrir et doué [...]). Ne pensez pas une minute que je vous propose cette galerie pour ne pas vous exposer aux "Cahiers d'Art". D'abord, nous ne faisons plus d'expositions à la galerie de la revue, puis cela vous priverait de la visite de critiques jaloux du succès des "Cahiers" [...] Il faut que vous profitiez de nos relations amicales avec Pierre, car à Paris les amitiés ne durent pas assez longtemps et la nôtre pour Pierre dure déjà depuis plus de quatre ans...». ${ }^{42}$

Nouvelle allusion également au travail de Picasso qui "fait des têtes magnifiques. Avant ces têtes, il a peint une série de tableaux dont quelques-uns sont reproduits dans le no 1-4, 1939 [Cabiers d'Art]. C'est vraiment un tour de force qui nous dépasse tous.". ${ }^{43}$

Selon Pierre Cabanne, les figures que peint Picasso à cette époque "sont le reflet de l'angoisse et de la peur" que 

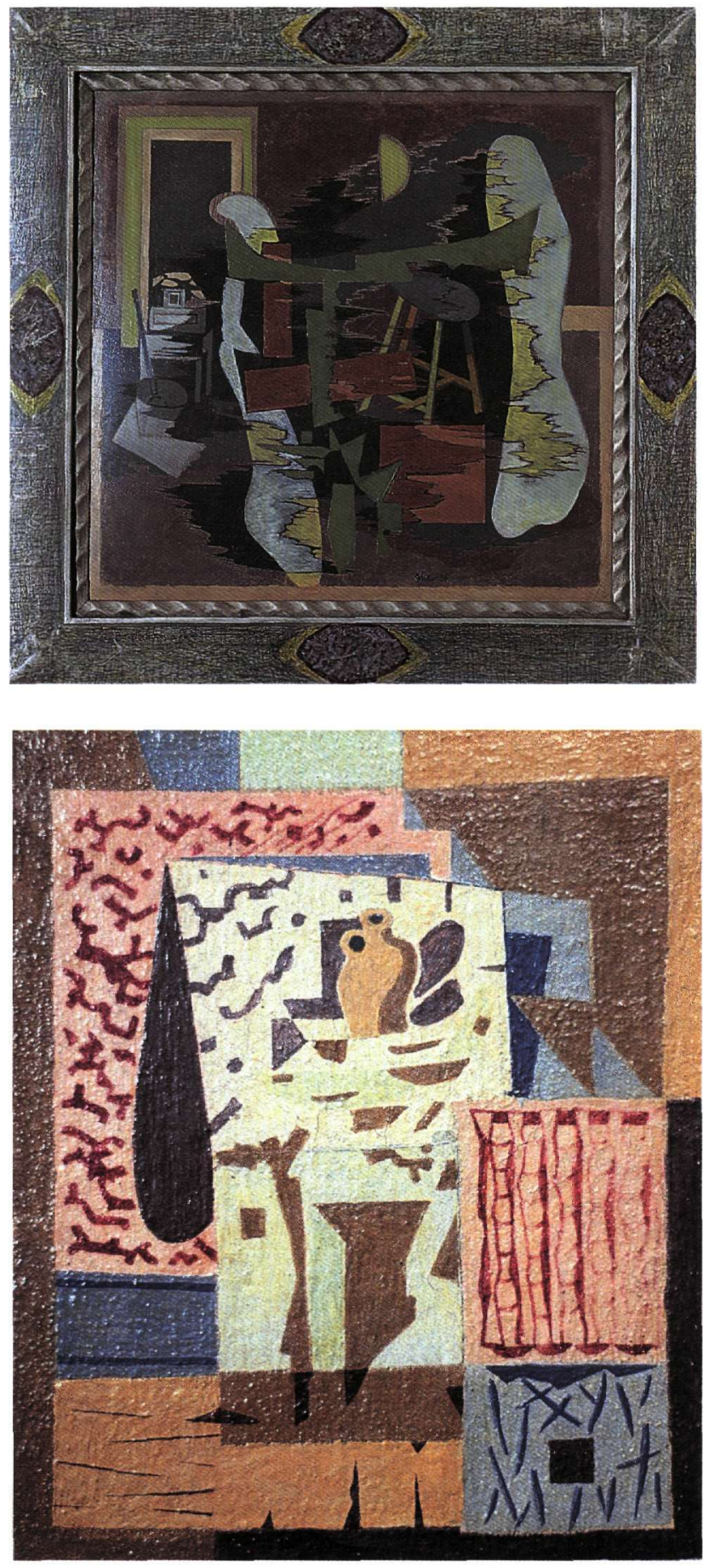

Fig. 6. N. Hadjikyriakos-Ghika, Deux formes (1936). Huile sur toile $(35$ x $35 \mathrm{~cm}$.). Musée Benaki, Pinacothèque Ghika.

Fig. 7. N. Hadjikyriakos-Ghika, Cruche en plein air (1938). Détrempe sur bois (22,5 x 19 cm.). Musée Benaki, Pinacothèque Ghika. provoque chez lui le climat de guerre qui envahit l'Europe. Peut-être les Zervos désignent-ils également ces têtes de taureau sur lesquelles l'artiste opère de nombreuses variations entre novembre 1938 et janvier 1939, violents dans la fragmentation des formes et dans l'éclat des couleurs. ${ }^{44}$

Yvonne Zervos quant à elle amplifie son activité créatrice malgré l'imminence de la guerre. Elle a monté "une affaire qui pourra la consacrer grand décorateur. [...] Depuis le temps, elle a fait d'énormes progrès comme architecte et comme "meublier" ", s'enorgueillit son mari. ${ }^{45}$

Cette "affaire" est une galerie qu'Yvonne Zervos a ouvert 12, rue Bonaparte à Paris, la galerie Mai. Selon Zervos, "elle est au point de vue de l'espace mural, plus grande que la galerie Paul Rosenberg..." Il ajoute que "la nécessité d'une galerie moderne se faisait sentir vu que la galerie Paul Rosenberg est fermée [à cause de ses origines juives?] et que Pierre Loeb ${ }^{47}$ est au front depuis le début des hostilités. [...] Elle donnera de la vie à Paris. [...]».

Face à l'adversité des temps présents, les Zervos tentent de réagir. "Nous travaillons tous deux comme si les événements n'existaient pas, autrement il faudrait se croiser les bras et crever d'ennui.».4.

Au seuil de la seconde guerre mondiale qui allait ainsi s'abattre sur la France pendant plusieurs années, Yvonne Zervos partage encore son enthousiasme et ses espoirs avec Tiggie. "...Depuis le 9 janvier 1940 j'ai ouvert ma galerie, non pas avec les "meubles" mais avec des tableaux et des sculptures [...]. Je suis heureuse au possible de faire cette galerie maintenant, car toutes sont fermées et tu ne peux t'imaginer combien on est heureux à Paris, que j'ai ouvert cette galerie, il y a énormément de visiteurs, tous les jours, ils viennent pour se donner du courage, cela les oblige à oublier ce "néant" des temps actuels. Tous les artistes s'enferment chez eux et travaillent magnifiquement [souligné dans le texte] bien. Leurs oeuvres deviennent encore plus belles ...». ${ }^{49}$

En ce contexte propice à certaines "réactions" de type culturel, ouvrir une galerie d'avant-garde n'était pas nécessairement du goût de tout le monde, comme le suggère l'anecdote rapportée par Yvonne à cette occasion. "...Ma galerie est grande! On peut y placer 40 tableaux assez grands. Elle est placée juste à côté des BeauxArts, aussi le lendemain du vernissage il y a eu au moins 200 élèves qui sont venus manifester [hostilité supposée des étudiants suivant une formation artistique de type 

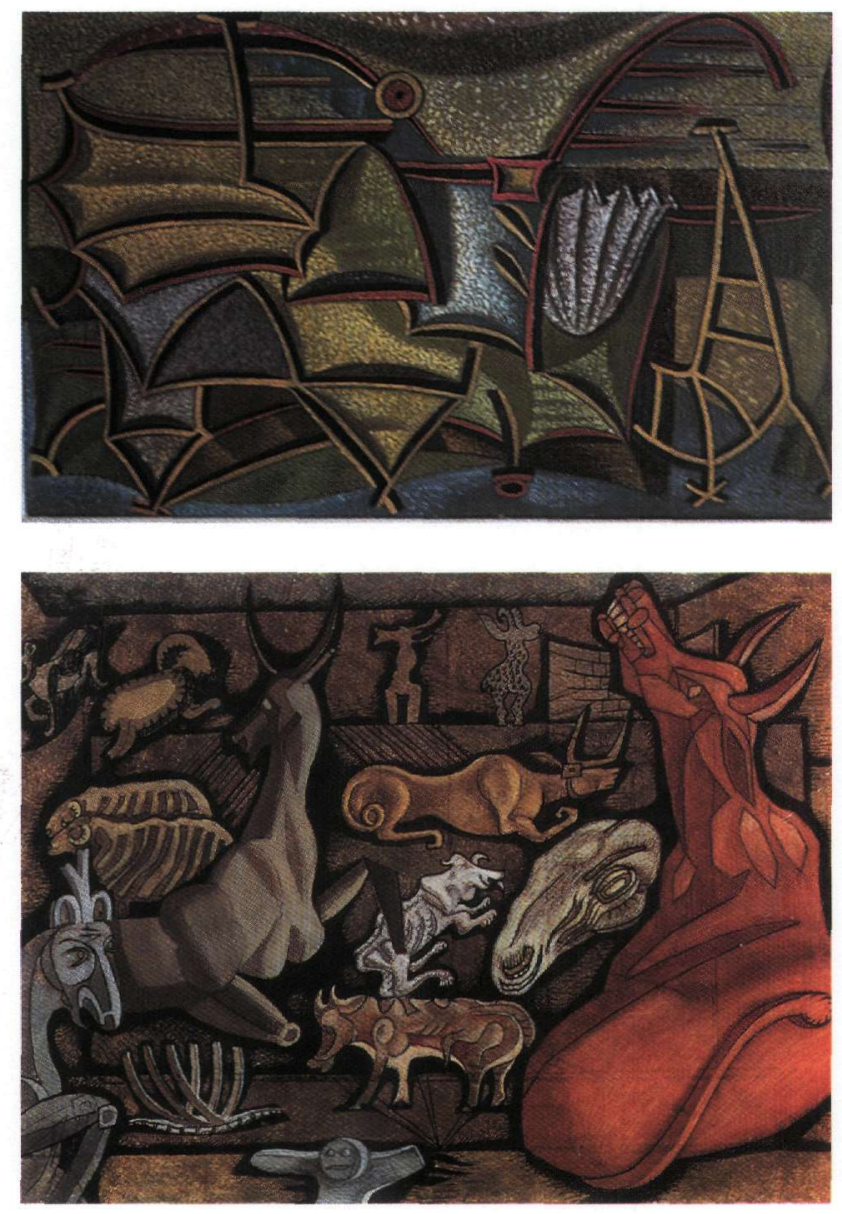

Fig. 8. N. Hadjikyriakos-Ghika, Paysage avec des tentes (1936). Huile sur bois $(23 \times 37 \mathrm{~cm}$.). Musée Benaki, Pinacothèque Ghika.

Fig. 9. N. Hadjikyriakos-Ghika, Animaux (1936). Huile sur carton $(27 \times 35 \mathrm{~cm}$.). Musée Benaki, Pinacothèque Ghika.

académique face à la modernité des oeuvres exposées], j'étais d'abord seule et j'ai eu très peur! pensant qu'ils casseraient tout. Mais je leur ai fait un discours, un discours incroyable pendant une heure et puis Zervos est arrivé et pour la première fois de ma vie, j'ai entendu Zervos leur parler encore pendant une heure. Si bien que nos discours ont porté et maintenant chaque jour ils viennent et regardent tout très attentivement. " ${ }^{50}$.

Et la dernière lettre d'Yvonne à Tiggie Ghika avant la fin du conflit s'achève sur ces paroles pleines de dignité. "...Je dois te dire que j'ai beaucoup d'espoirs encore! Je ne peux pas croire que toute l'humanité soit devenue complètement folle! J'ai confiance aux hommes ou plutôt à quelques êtres "bumains" pour trouver une solution afin d'empêcher une pareille destruction. [...] En tous cas si nous devons ne pas nous revoir d'ici longtemps sachez que je suis infiniment heureuse lorsque je pense à nous deux. Que ces quelques lignes vous apportent toute mon affection, toutes mes pensées [...]. Votre amie pour toujours...). ${ }^{51}$

NICOS HADJIKYRIAKOS-GHIKAS (1906-1994) LE PEINTRE Nico Hadjikyriakos-Ghika est né à Athènes le 26 février 1906 (figs 6-10). ${ }^{51}$ Il est le fils d'Alexandre Hadjikyriakos qui commanda la flotte grecque pendant la première guerre mondiale et fut plusieurs fois ministre, notamment sous le premier gouvernement républicain de Grèce dirigé par Eleuthérios Vénizélos. Sa mère, Hélène Ghika, appartient à une famille de souche albanaise dont certains membres participèrent au gouvernement de l'île d'Hydra et s'illustrèrent pendant la guerre d'indépendance de 1821 .

Ce qui caractérise principalement l'artiste est sa précocité. Dès l'âge de quinze ans, il suit les leçons de K. Parthénis (1878/9-1967), grand rénovateur de la peinture moderne en Grèce grâce à la synthèse de sa double expérience symboliste viennoise et moderniste parisienne. Après un premier séjour à Paris en 1919, Ghika s'installe progressivement dans la capitale française au cours des années vingt afin de suivre en parallèle une formation universitaire et artistique. Il y rencontre certains de ses compatriotes intellectuels, écrivains ou artistes, comme les critiques d'art Christian Zervos et Tériade, le sculpteur Michail Tombros et le poète Kostas Varnalis.

Sa première exposition personnelle remonte à 1927 (Paris, Gal. Percier, rue La Boétie). Ghika n'a encore que 21 ans. Picasso, qui s'y déplaça, crédita l'oeuvre du jeune artiste d'une grande "conscience morale". A partir de 1930, il intègre également les milieux artistiques d'avant-garde, fréquentant successivement les Léger, Laurens, Braque, Matisse, Lipschitz, Metzinger, Ozenfant et autres Le Corbusier, avec lequel il nouera une solide amitié et dont les recherches théoriques ne sont sans doute pas étrangères non plus aux premiers développements de son oeuvre. De retour dans sa patrie après 1934, il crée une revue, To trito mati ("Le troisième oeil"), qui condense ses réflexions sur la création contemporaine. Ghika peut alors être considéré comme le principal représentant théorique et plastique 
de la peinture grecque moderne d'inspiration cubiste ou post-cubiste.

Son oeuvre d'avant-guerre est essentiellement le reflet des nombreuses influences qu'il reçut lors de son apprentissage parisien, parmi lesquelles il faut citer en premier lieu celles de Léger, Matisse, Braque et Picasso. Il chercha cependant à adapter ces références à une vision personnelle de la création déterminée par la redécouverte des éléments plastiques constitutifs de son héritage culturel propre, la tradition hellénique antique et byzantine. Il rejoint en cela les préoccupations générales de la génération dite "des années trente", soucieuse de combiner les apports de la tradition artistique locale avec une ouverture aux développements récents proposés par l'Occident à partir de son centre parisien. Spéculative et violemment dirigée contre le romantisme "académique" d'inspiration bavaroise qui caractérisa une grande partie de la production artistique grecque jusqu'à la fin du dixneuvième siècle, l'oeuvre de Ghika participe de cette modernité "constructiviste" et volontariste, désireuse de représenter les choses non pas seulement telles qu'elles sont mais comme elles pourraient être, suivant ainsi une démarche que l'artiste qualifia lui-même de proprement "classique" et "méditerranéenne".

Progressivement cependant à partir de la fin des années trente et spécialement au lendemain de la seconde guerre mondiale, le peintre orientera son oeuvre dans une voie apparemment moins novatrice, marquée par un retour plus prononcé de la figuration, et une proximité plus grande par rapport aux éléments naturels de son environnement, dorénavant perçu autant à travers sa sensibilité que son intellect. Il s'éteindra le 3 septembre 1994.

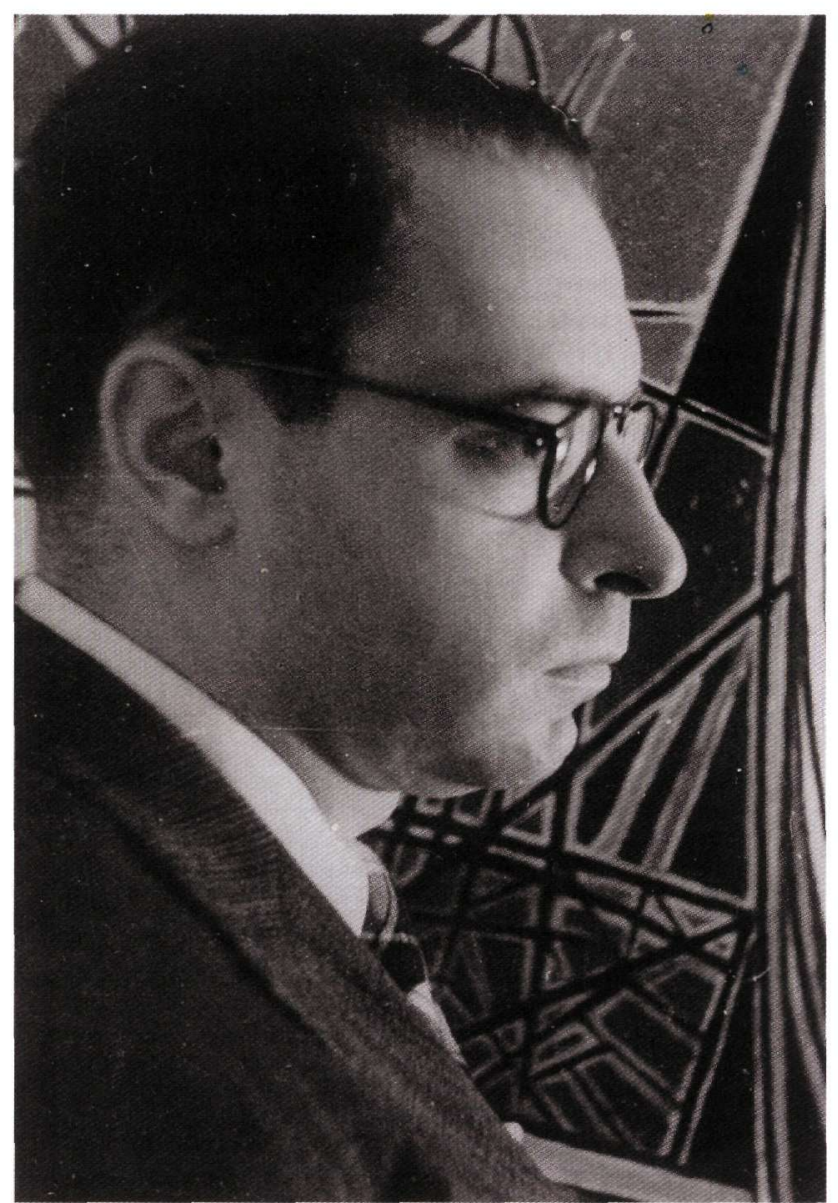

Fig. 10. Ghika. Photographié devant le tableau En regardant la chambre par la fénêtre (Mai-Juin 1933).

Jean-Pierre De Rycke

Docteur en Histoire de l'art

Université Catholique de Louvain (Belgique)

et Fondation A. Onassis (Athènes)

e-mail:jp_derycke@hotmail.com 


\section{N OTES}

* Cet article est le résultat d'un stage que j'ai effectué au Musée Benaki (Pinacothèque Ghika) entre le mois de novembre 2000 et le mois de janvier 2001. Je remercie chaleureusement Angelos Delivorrias, directeur du Musée Benaki, qui a permis son accomplissement; Ioanna Providi, responsable de la Pinacothèque Ghika, Alekos Zannas, Nikos Paisios et Ioanna Moraiti pour l'accueil, la confiance et l'appui qu'ils ont bien voulu me réserver, ainsi que Madame Evi Pavlov qui a eu la bienveillance de me proposer la présente publication.

1. Cette étude se limite à la première partie de la correspondance Ghika-Zervos qui couvre les années précédant la deuxième guerre mondiale. Interrompu pendant cette période, l'échange de lettres entre les Ghika et les Zervos reprendra à partir de 1945. Ce fond est toutefois moins dense et n'évoque plus que de façon épisodique la scène artistique parisienne.

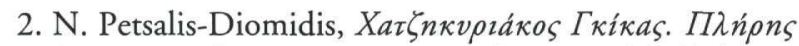

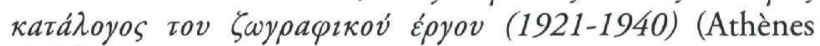
1979) 19.

3. Str. Eleutheriadis (dit "Tériade"), autre grand critique, éditeur d'art et promoteur de l'avant-garde parisienne dans l'entre-deux-guerres. Egalement grec d'origine, il fut le grand rival de Christian Zervos.

4. Sur le couple Zervos, v. notamment Hommage à Christian et Yvonne Zervos, Galeries Nationales d'Exposition du Grand Palais [Avignon], 11 décembre 1970 - 18 janvier 1971; R. Alberti, Picasso en Avignon (Paris 1971) 11-14.

5. V. spécialement la lettre du 3 août 1938 adressée par Yvonne Zervos à Tiggie Ghika.

6. Ch. Zervos, L'art en Grèce des temps prébistoriques au début du 18e siècle (Paris 1934).

7. V. lettre de Zervos à Ghika, non datée [1932-1933].

8. Cabiers d'art, 8-10 (1932).

9. V. Ch. Zervos, ibid.

10. Lettre de Zervos à Ghika, non datée [1934].

11. Tériade, Ecrits sur l'art (Paris 1996) 27.

12. V. Cahiers d'art, 5-6 (1935).

13. Sur les événements de février 1934, v. not. R. Remond, Notre siècle (1918-1991) (Histoire de France 6, Paris 1991) 160-66.

14. Lettre de Zervos à Ghika, non datée [février-mars $1934]$.

15. Il s'agit d'une "Composition" de 1934 actuellement conservée au Solomon R. Guggenheim de New York.

16. V. l'entête de la lettre d'Yvonne Zervos à Tiggie [Antigoni] Ghika du 26 mars 1935.
17. Lettre d'Yvonne Zervos à Tiggie Ghika du 26 mars 1935.

\section{Ibid.}

19. Zervos est notamment l'auteur du catalogue raisonné de Picasso qui comprendra finalement trente-trois volumes et représente de ce fait une référence incontournable pour la connaissance du peintre espagnol.

20. Lettre d'Yvonne Zervos à Tiggie Ghika, datée du 3 décembre 1935.

21. Ibid.

22. Ibid.

23. P. Cabanne, Le siècle de Picasso, I. La jeunesse, le cubisme, le théatre, l'amour (1881-1937) (Paris 1975) 472.

24. Ibid.

25. Ibid.

26. Ibid, 467.

27. Lettre d'Yvonne Zervos à Tiggie Ghika du 3 août 1938.

\section{Ibid.}

29. V. not. la lettre du 3 août 1938 et une lettre non datée que l'on peut situer en 1939.

30. Carte postale de Christian et Yvonne Zervos au couple Ghika, non datée [1936].

31. Lettre d'Yvonne Zervos au couple Ghika datée du 1er janvier 1937.

32. Ibid. Sur le problème des destructions occasionnées au patrimoine espagnol durant la guerre civile, v. ég. Ch. Zervos, Le sort des oeuvres d'art en Espagne Républicaine, Cahiers d'art 3-10 (1938) 212-28.

33. Lettre non datée de Zervos à Ghika [1939].

34. Ibid.

35. Alberti (n. 4) 12.

36. Ibid.

37. Ibid.

38. Ch. Zervos, Ghika (Paris 1965) 74.

39. Lettre d'Yvonne Zervos à Nico et Tiggie Ghika, 1er janvier 1937.

40. Lettre de Zervos à Ghika, non datée [1939].

41. Il s'agit de la galerie Pierre Loeb, inaugurée en 1924, rue Bonaparte.

42. Lettre de Zervos à Ghika, non datée [1939] (n.40).

43. Ibid. 
44. Cabanne (n. 23) 37.

45. Lettre de Zervos à Ghika [1939] (n. 40).

46. Lettre de Zervos au couple Ghika, 5 janvier 1940.

47. Détenteur de la galerie Pierre (v. supra).

48. Lettre du 5 janvier 1940 (n. 46).

49. Lettre d'Yvonne Zervos à Tiggie Ghika du 18 janvier 1940.
50. Ibid.

51. Ibid.

52. Pour de plus amples informations sur Ghika, v. not. Ch. Zervos, Ghika (Paris 1965); N. Petsalis-Domidis, Xa-

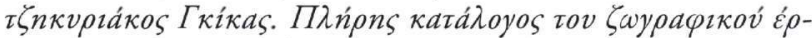
yov (1921-1940) (Athènes 1979); Ghika (Athènes 1991).

\section{JEAN-PIERRE DE RYCKE}

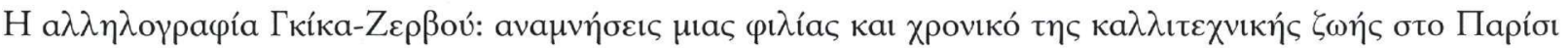

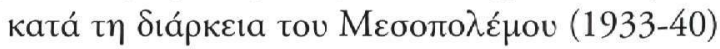

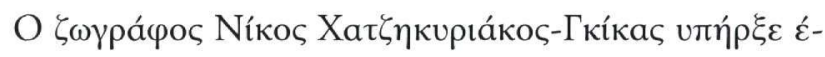
vaৎ a

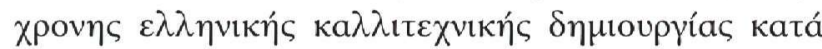

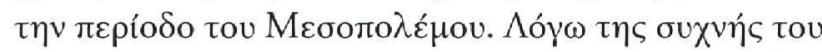

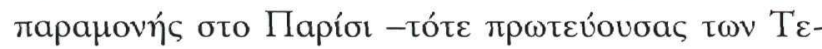

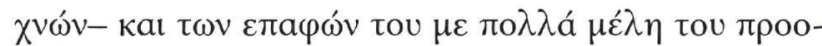

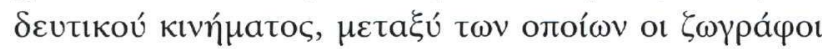

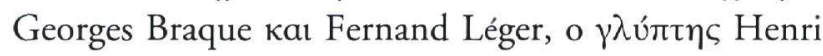

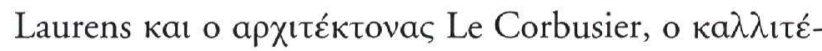

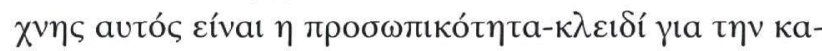

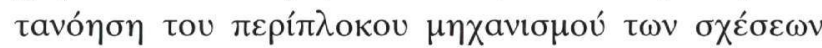

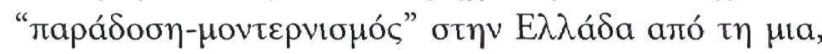

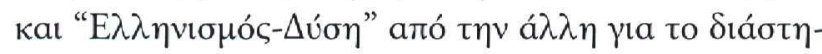
$\mu$ a autó.

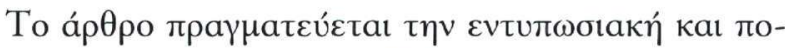

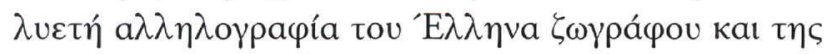

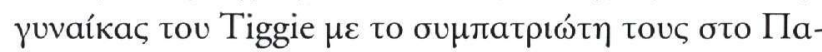

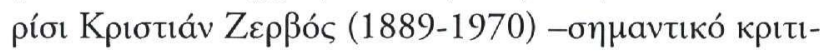

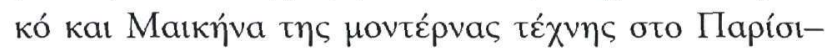

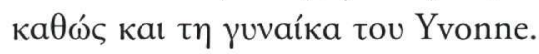

Пદ́pa a

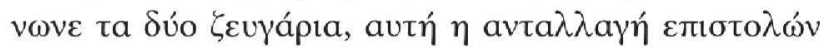

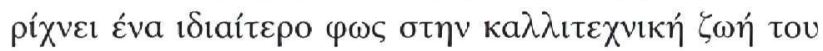

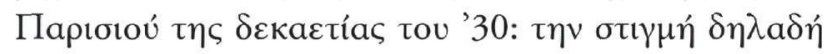

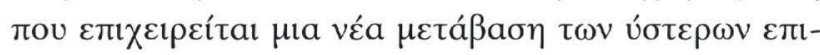

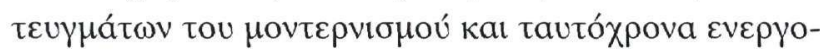

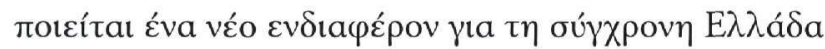

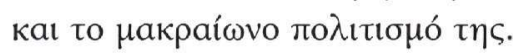

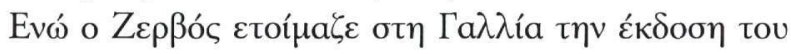

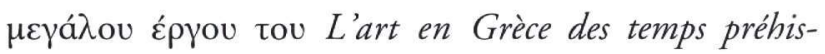

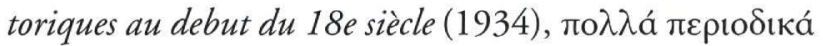

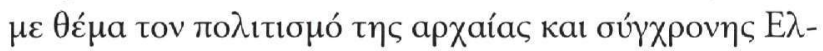

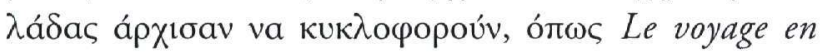

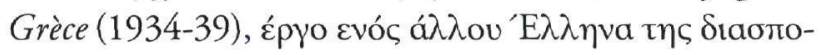

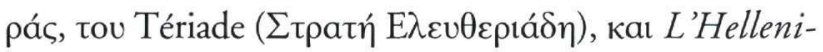
sme contemporain (1935-56).

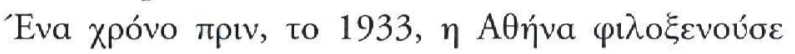

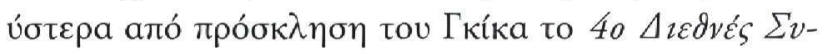

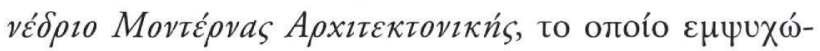

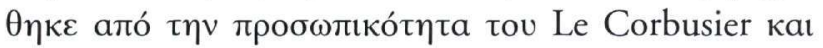

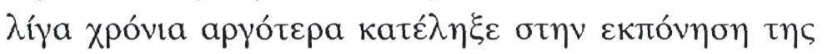

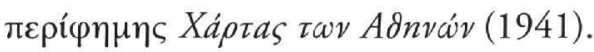

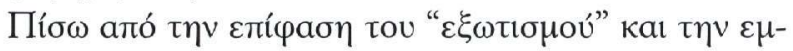

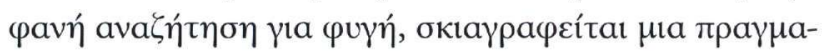

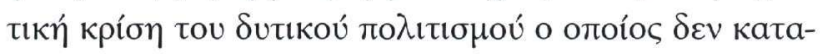

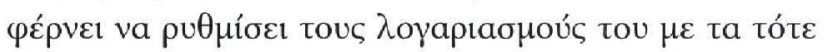

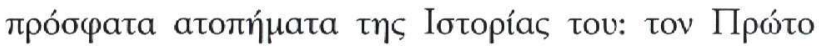

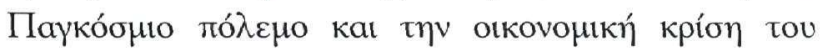
1929. Autó to $\delta ı \lambda \lambda$ ó́ $\mu \beta \beta$

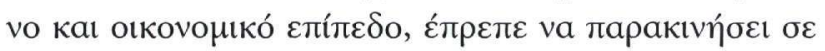

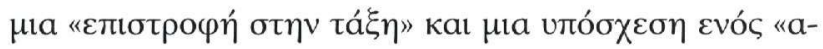

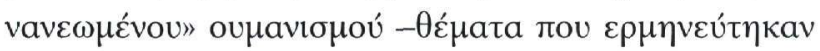

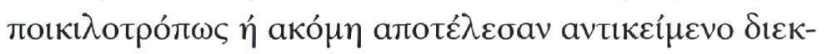

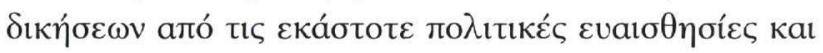

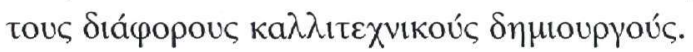

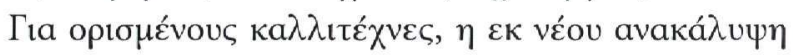

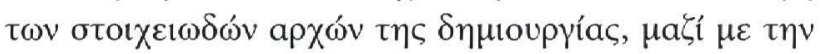

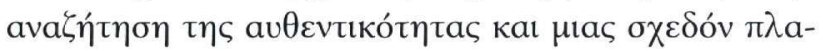




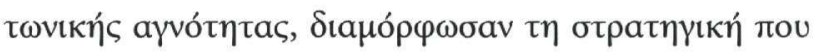

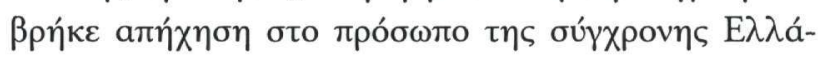

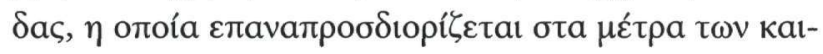

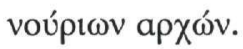

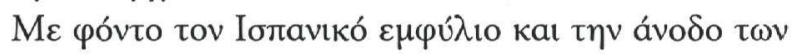

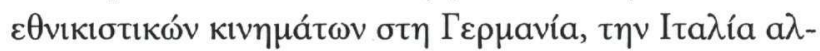

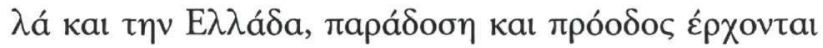

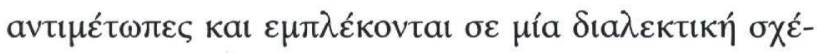

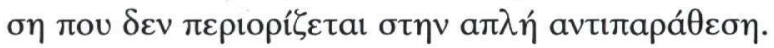

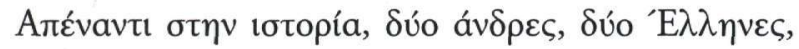

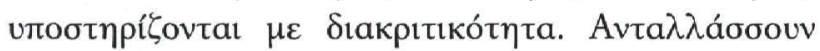

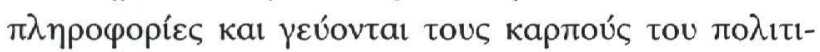

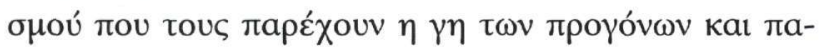

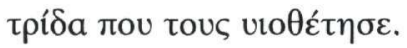

